\title{
Sprich (nicht) drüber - Fürsorgearbeit von Migrantinnen in deutschen Privathaushalten
}

Viele deutsche Haushalte beschäftigen eine Migrantin, die ihre Wohnung putzt, die kocht, wäscht, bügelt, die Kinder betreut, alte und schwache Angehörige versorgt. Dies erfolgt meistens ohne Arbeitsvertrag. Er wird durch mündliche Absprachen und Vertrauen ersetzt. Ohne die Weitergabe von Kontakten und Preisabsprachen über Netzwerke und ohne die weltweite ökonomische Ungleichheit, die dazu führt, dass (gut ausgebildete) Migrantinnen aus Osteuropa, Lateinamerika, Afrika und Asien mit Haushaltsarbeit bei uns mehr verdienen können als mit dem erlernten Beruf in ihren Herkunftsländern, würde dieser Arbeitsmarkt in der Grauzone nicht funktionieren. Der Beitrag beleuchtet die Akteurs- und Interessenkonstellationen im Bereich der haushaltsbezogenen Dienstleistungsarbeit.

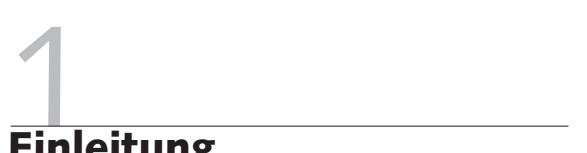

\section{Einleitung}

\subsection{FÜRSORGEARBEIT}

Haushaltsbezogene Dienstleistungsarbeit kann als Arbeit definiert werden, die im Privathaushalt erbracht wird und alle Tätigkeitsbereiche umfasst, die im englischen mit „Care-Work“ bezeichnet werden: Betreuung, Versorgung, Erziehung, Pflege von Menschen und Dingen, Unterstützung und Beratung. „Fürsorgearbeit“ als adäquate deutsche Übersetzung hat sich bislang nur in der wissenschaftlichen Debatte durchgesetzt, der Siebte Familienbericht (BMFSFJ 2006) spricht von „Familienarbeit“. Bei beiden Begriffen handelt es sich um eine vergeschlechtlichte Kategorie von Arbeit. Die im 19. Jahrhundert entstandene bürgerliche Geschlechterordnung (Bock/Duden 1977) etablierte die gesellschaftliche Trennung in privat und öffentlich getrennte Sphären; sie legte den Grundstein dafür, dass Erwerbsarbeit als männlich und Fürsorgearbeit als weiblich und diese Differenzierung zudem als "natürliche Ordnung“ codiert wurde. Mit dieser geschlechtsspezifischen Differenzierung ist gleichzeitig eine hierarchische Distinktion verbunden, bei der Berufsarbeit hoch bewertet und Fürsorgearbeit trivialisiert wird. Dieser Dualismus hat zwar eine Reihe von Wandlungen durchlaufen, ist aber als bürgerliches Familienideal keineswegs verschwunden.

\subsection{VERÄNDERTE RAHMEN- BEDINGUNGEN}

Als Folge eines emanzipativen Diskurses wird mittlerweile die „natürliche“ traditionelle geschlechtliche Arbeitsteilung infrage gestellt und Frauen der Weg in die Berufstätigkeit nahegelegt. Bis vor Kurzem ist allerdings - zumindest in der politischen Arena - kaum erörtert worden, von wem die weiterhin notwendige Familienarbeit verrichtet werden soll.

In Deutschland hat sich mittlerweile wie in anderen westlichen Ländern - eine Reihe von informellen Lösungen etabliert; eine davon ist das Weiterreichen (Outsourcing) von Fürsorgearbeit an eine im Privathaushalt tätige Person; historisch gesehen vollzieht sich damit die Wiedereinführung von Erwerbsarbeit in den spätmodernen Haushalt.

$\mathrm{Zu}$ den vielfältigen Gründen für diese Entwicklung gehört neben der - politisch gewollten - steigenden Erwerbstätigkeit von Frauen auch die Diversifikation von Familienformen (patchwork-Familien). Des Weiteren sind die zunehmende Eigenständigkeit der Lebensführung der Generationen und damit fehlende familiäre Unterstützungsnetzwerke wesentliche Faktoren. Darüber hinaus steigt das Lebensalter und damit der Anteil älterer Menschen an der Gesamtbevölkerung; für den sich daraus ergebenden umfangreichen Pflegebedarf steht bislang kein adäquates Angebot zur Verfügung. Auch Veränderungen des Arbeitsmarktes, etwa steigende Mobilitätsund Flexibilitätsanforderungen, die sich nur schwer mit verlässlichen Betreuungsarrangements verbinden lassen, sowie neue Anforderungen an die Familien, etwa Ex- pertenwissen in Bezug auf Gesundheit, Erziehung, Bildung, Freizeit- und Zeitmanagement etc., führen zu Situationen, in denen vor allem für Routinearbeiten im Haushalt Entlastung gesucht wird. Erschwerend kommt hinzu, dass eine Umverteilung von Erwerbs- und Familienarbeit zwischen den Geschlechtern nicht stattgefunden hat (Statistisches Bundesamt 2003). Zwar ist ein leichter Anstieg der männlichen Partizipation bei der Kinderbetreuungsarbeit zu verzeichnen, insgesamt jedoch intensivieren Männer ihr Engagement in der Berufsarbeit nach der Geburt von Kindern. Diese sogenannten Retraditionalisierungstendenzen ergänzen die Fortschreibung der „Schieflage der Geschlechtszuständigkeit“ (BMFSFJ 2006, S. 157) bei der Familienarbeit.

Die insgesamt in der gesamten westlichen Welt $\mathrm{zu}$ verzeichnende fallende Arbeitsmarktpartizipation von Männern (OECD 1999, S. 20) hat also keineswegs automatisch zu einer Umverteilung oder gar Gleichverteilung von Erwerbs- und Fürsorgearbeit geführt.

\subsection{BEGRIFFSDEFINITIONEN}

In der deutschen Debatte werden haushaltsnahe personenbezogene Dienstleistungen unterschieden nach sachbezogenen

Helma Lutz, Prof. Dr., (apl.) Universität
Münster. Arbeitsschwerpunkte: Geschlechter-
und Migrationsforschung, Intersektionalitäts-
und Ethnizitätsforschung, Biografie-
forschung.
e-mail: hlutz@uni-muenster.de

Helma Lutz, Prof. Dr., (apl.) Universität und Ethnizitätsforschung, Biografiee-mail:hlutz@uni-muenster.de 
Haushaltsarbeiten (Kochen, Waschen, Reinigung, Wäschepflege etc.) und personenbezogenen sozialen Betreuungs- und Pflegeleistungen, die Kindern, alten und kranken Menschen zugute kommen. Die angelsächsische Debatte dagegen fasst diese Tätigkeiten zusammen in den sogenannten drei C's: Cooking, Caring, Cleaning (Anderson 2000) und verdeutlicht damit gleichzeitig, dass in der privaten Dienstleistungsarbeit vielfach Kombinationen und Verschränkungen dieser Arbeitsfelder zu finden sind. So ist etwa in der gängigen Praxis das Kochen einer Mahlzeit für ein Kleinkind oder einen pflegebedürftigen Menschen eng mit der Hilfe bei der Nahrungsaufnahme verbunden, sodass die säuberliche Trennung der Bereiche eher dem Wunsch nach professionstechnisch nützlichen Ordnungskategorien als den Alltagsanforderungen zu entsprechen scheint. In der Fürsorgearbeit der drei C's ist ebenfalls sogenannte ,emotionale Arbeit" enthalten, etwa Geduld, Empathie, Zuneigung, also Anteile, die schwer messbar sind und doch gleichzeitig als Voraussetzung für die erforderlichen Tätigkeiten gelten. Begrifflich steht „Fürsorgearbeit" folglich für eine Vielzahl von unterschiedlichen physischen und psychischen Aktivitäten, die vorrangig im Privathaushalt (unentgeltlich und vorwiegend von Frauen) erbracht werden.

\section{Das Weiterreichen von Fürsorgearbeit}

Über den Umfang des „Outsourcing“ von Fürsorgearbeit gibt es bislang keine gesicherten Zahlen. Die Autorinnen und Autoren des Siebten Familienberichts (BMFSFJ 2006, S. 159) gehen zwar davon aus, dass der Privathaushalt heute der Beschäftigungssektor mit dem höchsten Anteil ungeschützter Arbeitsverhältnisse ist, belegen diese These jedoch nicht durch genauere Informationen. Bislang fehlen groß angelegte Erhebungen zum Bedarf und der tatsächlichen Inanspruchnahme von Dienstleistungen im Haushalt. Über die Gründe dafür kann nur spekuliert werden: Da es sich um einen Arbeitsmarkt in der Grauzone handelt, der schwer kontrollierbar und zudem im nicht-öffentlichen Bereich angesiedelt ist, scheint allgemein ein großes Unbehangen zu bestehen, diesen Bereich genauer zu untersuchen und/oder hier po- litisch zu intervenieren. ${ }^{1}$ Die Zahlen, die das Deutsche Institut für Wirtschaftsforschung (DIW) ermittelt hat, sind widersprüchlich: Ging man im Jahr 2005 noch davon aus, dass ca.10,3\% aller Bundesbürger (un-)regelmäßig eine Haushaltshilfe beschäftigen (DIW 2005), so wird für das Jahr 2006 gemeldet, dass der Bedarf sich auf ca. $4 \%$ beläuft (Schupp et al. 2006). Erklärt wird dieser Rückgang nicht. Bei der Interpretation der Daten muss jedoch berücksichtigt werden, dass das DIW eine bestimmte Argumentationslinie vertritt: Gerade in diesem Bereich wird ein großes Beschäftigungspotenzial für Langzeitarbeitslose gesehen, und diese Vorstellung wurde in entsprechenden arbeitspolitischen Maßnahmen umgesetzt. ${ }^{2}$ Auch ist in den vergangenen Jahren das Modell der Dienstleistungspools propagiert worden, das die erforderlichen Leistungen legal anbietet; bislang hat sich diese Lösung allerdings nicht durchsetzen können, unter anderem, weil Kunden nicht bereit sind, die hohen Preise zu bezahlen (Weinkopf 2002) und weil durch die Pools eine personenbezogene Kontinuität und Flexibilität, etwa bei der Betreuung von Kindern und pflegebedürftigen Personen, nicht unbedingt gewährleistet werden kann.

Aus den bisherigen Ausführungen ist abzuleiten, dass in jüngster Zeit zum einen arbeitsmarktpolitische Anstrengungen unternommen wurden, um Arbeitsplätze im Haushalt für deutsche Arbeitslose zu schaffen; zum anderen - damit unmittelbar zusammenhängend - besteht offenbar wenig Interesse daran, offiziell zur Kenntnis zu nehmen, dass Fürsorgearbeit sich zu einem umfangreichen Arbeitsmarkt für Migrantinnen entwickelt hat. ${ }^{3}$

\section{Migrantinnen in der Fürsorgearbeit}

\subsection{ZWISCHEN ILLEGALEM STATUS...}

Eine wachsende Anzahl von Publikationen, unter anderem eine von mir in drei Städten (Berlin, Hamburg und Münster) durchgeführte Studie (Lutz 2007a), belegt mittlerweile, dass sowohl im urbanen als auch im ländlichen Bereich der Anteil bezahlter Haushaltsarbeiterinnen zunimmt und dass es sich hierbei vorrangig um Migrantinnen aus Osteuropa, Lateinamerika, aber auch aus Asien und Afrika handelt (Rerrich 2006; Alt 2003; Anderson 2003; Shinozaki 2003; Hess 2005). Diese Frauen sind sowohl in der Kinderbetreuung und vor allem beim Verrichten von Putz- und Pflegearbeiten tätig als auch in Haushalten alter und pflegebedürftiger Menschen (Anonymus 2007; Holch 2006).

Die - vorrangig qualitativen - Studien weisen darauf hin, dass diese Tätigkeiten nicht legal erbracht werden, also nicht durch einen Arbeitsvertrag abgesichert sind, da Migrantinnen für diese Tätigkeit keine Arbeitserlaubnis erhalten und darüber hinaus oft aufenthaltsrechtlich illegal sind. Die meisten Migrantinnen, die in diesem Sektor arbeiten, reisen zwar legal mit einem Touristen- oder zu Studienzwecken erteilten Visum ein, sind aber nach Ablauf dieser Fristen gezwungen, sich in einem Leben als Illegale zurechtzufinden. Illegaler Aufenthalt gilt mittlerweile als Straftatbestand, der mit Ausweisung sanktioniert wird. ${ }^{4}$

1 Dafür spricht auch, dass ein Gesetzentwurf des Finanzministeriums, in dem die illegale Beschäftigung von Reinigungskräften und Babysittern im Privathaushalt zum Strafbestand erklärt werden sollte, nach Protesten von Abgeordneten aller Parteien zurückgezogen wurde. Bis auf Weiteres gilt diese Beschäftigung als Ordnungswidrigkeit und nicht als Straftatbestand.

2 Das DIW ging im Jahr 2003 davon aus, dass im Bereich der haushaltsnahen Dienstleistungen 500.000 neue Jobs für schwer zu vermittelnde ungelernte und ältere Arbeitnehmer geschaffen werden können, wenn einerseits potenzielle Arbeitgeber dazu steuerlich animiert werden (Zimmermann 2003, S. 18) und andererseits Hartz IV-Empfänger und Langzeitarbeitslose mithilfe von Einschränkungen von Leistungen zur Aufnahme dieser Tätigkeiten gezwungen würden (ebd., S. 22). Die „Deregulierung von Dienstleistungsmärkten insbesondere im Gesundheitswesen, bei der Pflege und der Altenund Kinderbetreuung " sollte ein wichtiges Ziel und Instrument der Arbeitsmarktreform sein (ebd.). Obgleich es bereits Hinweise gab, dass diese Projektion von falschen Voraussetzungen ausging, etwa der Annahme, dass es sich um leicht zu erlernende und durchzuführende Arbeit handele (Weinkopf 2003), wurden die DIW-Ideen in das Hartz-Gesamtwerk aufgenommen. Drei Jahre später bestätigt sich die Skepsis und zeigt sich, dass neue Arbeitsverhältnisse aufgrund der Mini-JobRegelung kaum entstanden sind (Schupp et al. 2006).

3 Das politische Diktum, dass Deutschland keine Einwanderung im Bereich der Niedriglohnarbeit benötigt, bleibt hier dominant.

4 Die Schätzungen der Gesamtzahl aller aufenthaltsrechtlichen Illegalen in Deutschland schwanken zwischen 500.000 und 1,5 Mio. (Cyrus 2004); mindestens die Hälfte von ihnen sind Frauen. 


\section{2 ...UND GRAUZONEN- BESCHÄFTIGUNG}

Die einzige „Greencard“ in diesem Bereich wurde für „Haushaltshilfen in Haushalten mit Pflegebedürftigen" geschaffen, die aus Osteuropa rekrutiert und über die Zentralstelle für Arbeitsvermittlung (ZAV) vermittelt werden und für eine Höchstdauer von drei Jahren in dem Haushalt, für den sie angefordert wurden, arbeiten können. Die Arbeitszeit beträgt offiziell 38,5 Stunden, und im Prinzip dürfen diese Frauen keine medizinischen und pflegerischen Leistungen erbringen, selbst dann nicht, wenn sie im Gesundheitsbereich ausgebildet sind; für die Pflege sind weiterhin ambulante Pflegedienste deutscher Anbieter zuständig. In der Praxis wird die vorgesehene Arbeitszeit kaum eingehalten, da es sich bei dem Beschäftigungsarrangement in den meisten Fällen um ein Live-In-Arbeitsverhältnis handelt; das bedeutet, dass die betreffende Arbeitnehmerin im Haushalt wohnt und tendenziell rund um die Uhr zur Verfügung steht. Für Arbeitgeber ist insbesondere das Standardgehalt von $1.250 €$ attraktiv, ${ }^{5} \mathrm{da}$ eine entsprechende Betreuung durch eine deutsche ausgebildete Krankenschwester oder Pflegehelferin zwischen 5.000 und $8.000 €$ (Holch 2006) kostet. Erstaunlicherweise gibt die ZAV nun an, im Jahr 2006 insgesamt lediglich 1.700 Anträge bearbeitet zu haben, während andere Quellen (Anonymus 2007) davon ausgehen, dass ca. 100.000 vorwiegend aus Osteuropa stammende Frauen als LiveIn in der Rundum-Pflege beschäftigt sind. Es ist zu vermuten, dass die legale ZAVLösung für viele Arbeitgeber unattraktiv ist, weil sie einen hohen Verwaltungsaufwand erfordert und für Angehörige, die nach einer schnellen Lösung der Hauspflege suchen, zu langwierig und wenig passfähig ist; ebenso wenig attraktiv ist sie für die betroffene Arbeitnehmerin, da nach Abzug von Beiträgen zur Arbeitslosen-, Sozial-, Pflege- und Rentenversicherung sowie Kost und Logis je nach Aushandlung 560 bis $1.000 €$ übrig bleiben (Lutz 2007a, S. 175f.).

Für die betroffenen Migrantinnen haben sich nun im Zuge der EU Erweiterung nach Osteuropa im Jahr 2004 zusätzliche Möglichkeiten zur Arbeitsaufnahme eröffnet, die finanziell für sie attraktiver zu sein scheinen. Obwohl die BRD den Arbeitsmarkt für osteuropäische EU-Arbeitnehmer bis zum Jahr 2011 geschlossen hat, gibt es zahlreiche Möglichkeiten, über die „Freizügigkeit der Dienstleistung" (2006/123/ EG) innerhalb der EU nach Deutschland zu kommen; so können etwa „Haushaltshilfen " und selbst qualifizierte Pflegekräfte in Deutschland ohne die Zustimmung durch die Bundesagentur für Arbeit tätig sein, wenn sie

- eine vorübergehende Dienstleistung erbringen,

- ihren ökonomischen Schwerpunkt im Herkunftsland haben und

- der Sitz des sie entsendenden Unternehmens bzw. die Ich-AG im Herkunftsland ist (Richtlinie über Entsendung von Arbeitnehmern 96/71/EG).

Im Prinzip handelt es sich dabei also um eine Form der Leiharbeit, bei der ein osteuropäisches Unternehmen mit einer deutschen Vermittlungsagentur zusammenarbeitet. Die Vermittlung erfolgt über das Internet und es werden entweder einmalig (bis zu $800 €$ ) oder monatlich zu zahlende Gebühren erhoben; der Nettoverdienst liegt zwischen 1.000 und $1.500 €$.

Pflegeverbände haben durch diesen neu entstandenen Markt - wie befürchtet eine Konkurrenz von Billiganbietern bekommen, die sich weitgehend in einer Grauzone bewegen, denn bislang wird die Einhaltung der rechtlichen Voraussetzungen für eine solche Tätigkeit (etwa die Sozialversicherungszahlungen der Leiharbeitsunternehmen im Herkunftsland) nicht überprüft. In einer von mir geleiteten, laufenden Studie ${ }^{6}$ zeichnet sich ab, dass sowohl die Arbeitgeberinnen bzw. Arbeitgeber als auch die betroffenen Migrantinnen kaum in der Lage sind, die Legalität des Arbeitsverhältnisses zu beurteilen, und es gibt bislang für beide Seiten keinerlei Ansprechpartner, die Rat und Hilfe leisten können und bei eventuellen Missständen intervenieren.

Ein vergleichbares Live-In-Arbeitverhältnis lässt sich auch in der Kinderbetreuung durch Au-Pairs finden. ${ }^{7}$ Daneben existiert weiterhin ein zahlenmäßig weit umfangreicherer Sektor von migrantischen Live-Outs, also Frauen, die täglich mehrere Haushalte putzen, Kinder betreuen, Einkäufe für alte Menschen erledigen etc. Die Vermittlung erfolgt in diesem Bereich auf beiden Seiten über Freundes- und Verwandtschaftsnetzwerke, die Bezahlung erfolgt stunden- oder Tagesteil-weise. Sobald diese Frauen einen breiten Kundenkreis aufgebaut haben, können sie ihre eigenen Kriterien in Bezug auf die Auswahl ihrer Kunden aufstellen und dementsprechend eine Arbeitsplatzauswahl treffen. Das gelingt allerdings nur dann, wenn die Konkurrenz nicht zu groß ist und das sehr sensible, nicht durch einen Arbeitsvertrag, sondern durch Vertrauen regulierte Arbeitsverhältnis instandgehalten wird.

Aus meiner eigenen Untersuchung (Lutz 2007a), in der sowohl Arbeitgeberinnen bzw. Arbeitgeber als auch Migrantinnen befragt wurden, geht hervor, dass beide Seiten in der Regel (sehr) gut ausgebildet sind: Unter den Arbeitgebern ist der Anteil der akademisch Ausgebildeten zwar höher als bei den Arbeitnehmerinnen, jedoch fiel der hohe Bildungsabschluss auch bei Letzteren auf.

Die Hälfte der von uns befragten Migrantinnen hat Kinder im Heimatland zurückgelassen; verschiedene Gründe sind hierfür zu nennen: Zum einen haben Illegale weder ein Recht auf Gesundheitsversorgung noch auf Familienzusammenführung, und den Kindern fehlt das Recht auf (Schul-/Aus-)Bildung (Lutz 2007a, S. 169 ff.). Zum anderen gehen die betroffenen Mütter in der Regel davon aus, dass die Migration nicht auf Dauer angelegt ist, ${ }^{8}$ und sie bevorzugen Lösungen, in denen etwa durch Pendelmigration im drei-monatigen Rhythmus die Betreuung der Kinder nicht vollständig Familienmitgliedern oder familienfremden Personen überlassen werden muss. Für Osteuropäerinnen ist diese Form der Haushaltsführung in zwei Ländern besser zu realisieren als für Frauen aus Lateinamerika, Asien oder Afrika, die oft jahrelang abwesend sind, weil jede Rückkehr in das Heimatland mit dem Risiko verbunden ist, nicht mehr in Deutschland einreisen zu können.

\footnotetext{
5 Dies entspricht in etwa dem Betrag, den Angehörige von der Pflegeversicherung für die häusliche Betreuung von Pflegebedürftigen ab Pflegestufe Zwei erhalten.

6 Es handelt sich um ein von der Deutschen Forschungsgemeinschaft finanziertes europäisches Netzwerkprojekt: Landscapes of Care Drain. Care provision and Care Chains from the Ukraine to Poland and from Poland to Germany (2007-2010).

7 Zur Problematik dieser ursprünglich als Kulturaustausch betrachteten, mittlerweile aber zur Haushaltsarbeit mutierten Form des Live-In vgl. Hess 2005 und Lutz 2007a, S. 109ff.

8 Dennoch waren einige Frauen bereits länger als zehn Jahre in diesem Bereich tätig.
} 


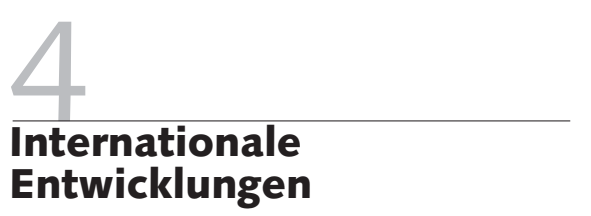

4.1 DIE POLITIK DER ZIELLÄNDER

Ähnliche Entwicklungen wie in Deutschland sind in den meisten (post-)industriellen Ländern zu verzeichnen (für Europa siehe Lutz 2007b). Neben Gemeinsamkeiten, etwa die niedrige gesellschaftliche und finanzielle Bewertung der Arbeit und vergleichbare Arbeitsplatzarrangements (Live-In versus Live-Out), lassen sich auch viele Differenzen feststellen. Unterschiede ergeben sich zum einen in der Frage des aufenthaltsrechtlichen Status (legal versus illegal): Viele Länder rekrutieren über Kontingente aktiv Migrantinnen für die Arbeit (in der EU sind dies vor allem die Mittelmeerländer Spanien, Italien und Griechenland, aber auch England und Irland) und/ oder eröffnen die Möglichkeit, über Legalisierungsregelungen ex post ein Aufenthaltsrecht zu erhalten. Damit wird in der Regel auch sukzessive ein Recht auf Niederlassung und Familienzusammenführung erworben. Zum anderen unterscheiden sich deutlich die jeweiligen nationalen Migrationsregime (Zulassung, Abweisung, Ignoranz), die letztendlich darüber entscheiden, ob den betreffenden Frauen ein potenzieller Platz in der Gesellschaft zugestanden wird oder nicht.

Internationale Studien belegen die Existenz eines Weltmarkts für Haushaltsarbeiterinnen, der sowohl auf der Seite der Zielals auch der Herkunftsländer über Anwerbe- und Vermittlungsagenturen sowie kollektive Netzwerke hochgradig organisiert ist (Momsen 1999, S. 5). Neben internationalen Vermittlungsagenturen, dem Internet und nationalen Rekrutierungsprogrammen kommen viele Vermittlungen durch private Kontakte, aber auch durch religiöse Organisationen zustande, etwa die katholische Kirche in Spanien und Italien (Andall 2000; 2003; Parreňas 2001).

Aus den internationalen Veröffentlichungen lässt sich ableiten, dass Haushaltsarbeit weltweit eine eher versteckte Angelegenheit ist (Andall 2003, S. 39), und zwar deshalb, weil der private Raum - trotz großer kultureller Unterschiede - eine Tabuzone für staatliche Kontrollen darstellt. Zunächst scheint diese Tatsache positive Auswirkungen zu haben, denn der Privat- haushalt als Arbeitsort wird auch von den betroffenen Migrantinnen als Schutzraum betrachtet. Andererseits ermöglicht die Segregation dieses Sozialraums die ungestrafte Ausübung von Gewalt und erleichtert Ausbeutung und Missbrauch. Der antizipierte Schutzraum kann also zum Gefängnis werden. Vor allem für Live-Ins gibt es das Dilemma, dass die Aufgabe des Arbeitsplatzes zugleich bedeutet, die Unterkunft zu verlieren.

Eine arbeitsrechtliche Regulierung fehlt in vielen Ländern, da Haushaltsarbeit als Familienarbeit betrachtet wird. Deswegen gibt es auch kaum Möglichkeiten, Missbrauch aufzuspüren und zu sanktionieren.

Bürgerrechte, wie etwa politische Rechte, werden in der Regel erst dann zugestanden, wenn die Migrantinnen die jeweilige Staatsbürgerschaft erworben haben. Auffällig sind jedoch auch Unterschiede, die beim Umgang mit Menschenrechten für aufenthaltsrechtlich Illegale in der EU zu finden sind (Cyrus 2004; Alt 2003; Anderson, B. 2000; Anderson, Ph. 2003): Im Gegensatz zu Ländern wie Spanien, Italien, den Niederlanden u.a. gibt es etwa in Deutschland keine Regelung für die Gesundheitsversorgung, und im Kontrast zu allen anderen Staaten der EU ist das Recht auf Bildung dadurch beschnitten, dass Beamte und Angestellte im öffentlichen Dienst eine Übermittlungspflicht haben; d.h. sie müssen den Ausländerbehörden melden, wenn Kinder ohne Aufenthaltsgenehmigung in Kindergärten oder Schulen angemeldet werden (Cyrus 2004).

Obgleich ein Leben als Illegale ungleich schwieriger zu bewältigen ist, findet sich selbst in Ländern, in denen die Rekrutierung von Haushaltsarbeiterinnen legal stattfindet, ein hoher Anteil an irregulären und undokumentierten Migrantinnen (für Europa: Andall 2003). Die Verheißung, in kurzer Zeit - aus dem Herkunftskontext gesehen - höhere Einkünfte zu erzielen, führt offensichtlich dazu, dass viele Frauen die damit verbundenen Risiken in Kauf nehmen. Kaum ein Aufnahmeland scheint daran interessiert, diese Situation grundlegend verändern zu wollen.

\subsection{DIE SITUATION DER HERKUNFTSLÄNDER}

Die Entsendeländer wiederum haben aufgrund ihrer geo-politischen Lage entweder nur begrenzten Einfluss, die Arbeitsrechte ihrer emigrierten Staatsbürgerinnen zu verbessern, oder aber sie sind auf die Milliardenbeträge der Remissionen ${ }^{9}$ dieser Migrantinnen, die einen wichtigen, teilweise sogar den wichtigsten Beitrag zum jeweiligen Nationaleinkommen leisten, so sehr angewiesen, dass sie eher Stillschweigen bewahren und nur in krassen Fällen eingreifen. Ihre Anstrengungen beziehen sich eher darauf, den emigrierenden Frauen die Wahrung emotionaler und finanzieller Loyalität zu ihrem Heimatland nahe zu legen. Und tatsächlich gilt: Wie ihre historischen Vorgängerinnen, die Dienstmädchen, scheint die Mehrheit dieser Frauen zurückgebliebenen Familienangehörigen (Ehemännern, Eltern, Kindern, Verwandten) eine größere Loyalität entgegenzubringen als emigrierende Männer (Momsen 1999; Pessar/Mahler 2003).

Da die Haushaltsarbeiterinnen heute in der Regel älter sind als ihre historischen Vorgängerinnen und nicht selten bereits eine eigene Familie gegründet haben, die sie zurücklassen müssen, entstehen zunehmend transnationale Haushalte und transnationale Mutterschaftsarrangements (Hondagnen-Sotelo/Avila 1997). In diesem Zusammenhang hat Arlie Hochschild (2000) den Begriff ,globale Betreuungskette" (global care chain) geprägt, der mittlerweile zu einem einflussreichen Konzept in der akademischen Auseinandersetzung über migrantische Hausarbeit avanciert ist. Gemeint ist damit, dass die ökonomische Schieflage zwischen hoch industrialisierten Ländern und denjenigen der „Dritten Welt" und Osteuropas der Kommodifizierung emotionaler, versorgender Arbeit ${ }^{10}$ und der Verstetigung geschlechtsspezifischer Ungleichheit bei der Verteilung reproduktiver Arbeit in den Zielländern Vorschub geleistet hat. In den Herkunftsländern wird zwar ökonomisches Kapital akkumuliert, gleichzeitig entsteht jedoch durch den Abzug von Versorgungskapital ein Versorgungsdefizit (care drain).

Diese Analyse scheint deshalb so wichtig, da für einige Entsendeländer die menschliche Ressource der einzige exportierbare „Rohstoff“ ist, den sie noch haben. Auch wenn die Weltbank diesen über Remissionen materialisierten Rohstoff mitt-

\footnotetext{
9 Geld, das als Rücküberweisung entweder über Banken oder über private Kuriere in das Herkunftsland geschickt wird.

10 Vermarktung von Reproduktionsarbeit.
} 
lerweile als den wichtigsten Beitrag zur Entwicklung der nationalen Ökonomien vieler sogenannter Entwicklungsländer betrachtet (Sørensen 2005), ist nicht zu übersehen, dass die migrierten Mütter und deren zurückgebliebene Kinder am unteren Ende dieser Kette stehen und oft den emotionalen und sozialen Preis für den Abzug der Versorgungsressourcen bezahlen.

Noch ein anderer Aspekt kommt hinzu: Da die Bindung zwischen Müttern und ihren Kindern aufgrund der oft jahrelangen räumlichen Trennung vorwiegend über die Versorgung für den Lebensunterhalt und eine gute Ausbildung in Privatschulen oder -universitäten zum Ausdruck kommt, entsteht eine „Mutterschaft auf Distanz" (Parreñas 2005), deren langfristige Folgen noch ungenügend erforscht sind.

Über die bereits genannten Befunde hinaus sind transnationale Dienstleistungen auch eine Folge der in den Ländern der „Dritten Welt" verbesserten oder, wie in Osteuropa, traditionell guten Bildungsund Kommunikationssituation sowie von verbesserten Reisemöglichkeiten oder Erleichterungen des Grenzübertritts in den Entsendeländern. Gleichzeitig verschlechtern sich oder stagnieren die dortigen Lebensbedingungen. Immer mehr Frauen sind stärker geneigt zu migrieren und verlassen ihre Länder nicht so sehr trotz, sondern wegen ihres erhöhten Bildungskapitals, das in den Herkunftsregionen keine angemessene Verwendung (mehr) findet, ihnen aber den Weg ins Ausland erleichtert. Auffällig ist auch, dass die mit der Migration verbundenen Probleme oft nicht antizipiert, sondern im Gegenteil verdrängt werden, was möglicherweise auch eine Erklärung für die hohe Mobilitätsbereitschaft dieser Migrantinnen ist.

\section{Sprich (nicht) drüber: Die Umverteilung von Fürsorgearbeit auf Migrantinnen}

„Ja. Also ich würde das auch allen Frauen empfehlen (lacht) eher zu machen als in schrägen - schrägen Kleinfamilienstrukturen traditioneller Art zu verharren, ne. Ich glaube wirklich, dass das ein gutes Alternativmodell ist und für mich war's ein Befreiungs-befreiendes Modell, ne... mirgeht's jedenfalls heut bedeutend besser als vor 10 Jahren oder so, ja. Und ich finde, das ist wirklich ne Alternative. Abgesehen davon, dass es ne Arbeitsbeschaffungsmaßnahme ist und ne Dienstleistungsmaßnahme" (Lutz 2007a, S. 112).

Mit diesen Worten bilanziert die Arbeitgeberin Ursula Pelz ${ }^{11}$ das mehr als zehnjährige Zusammenleben mit der Ukrainerin Tamara Jagellowsk. Tamara kam als Neunzehnjährige zu ihr als Au-Pair und betreute vom ersten Tag an ihre beiden damals noch kleinen Söhne. Ursula Pelz lebte getrennt von ihren Mann, hatte gerade eine Vollzeitstelle aufgenommen, arbeitete parallel an ihrer Promotion und machte anschließend Karriere im Wissenschaftsbetrieb. In all den Jahren hat Tamara, deren $\mathrm{Au}$-Pair-Status durch immer wieder neue Aufenthaltsarrangements (als Sprachenschülerin, Studienkollegiatin, Studentin, Partnerin etc.) abgelöst wurde, sie von der Fürsorgearbeit weitgehend entlastet: Kochen, Putzen, Aufräumen, die Betreuung der Kinder im Alltag etc. Geblieben ist das Live-In-Arrangement mit der Entlohnung eines Taschengeldes. Die mittlerweile 30jährige Tamara hat in der Zwischenzeit ausgezeichnet Deutsch gelernt, aber keine einzige Ausbildung abgeschlossen. Sie liebt die Kinder, sieht sich als soziale Mutter, ist stolz auf ihre Erziehungsleistungen. Jedoch fällt ihre Bilanz - anders als die von Ursula Pelz - nicht positiv aus. Im Vergleich mit ihren Freundinnen in der Ukraine fühlt sie sich als Versagerin (Lutz 2007a, S. 113).

Das Alternativmodell, das von Frau Pelz als Gegenentwurf zum „schrägen Familienmodell“ gepriesen wird, entpuppt sich bei näherer Betrachtung als ein genderkonformes Ersatzmuster, bei dem Ursula Pelz die Rolle der Familienernährerin übernommen hat und Tamara Jagellowsk die der klassischen Ehefrau, die ihre eigenen beruflichen Ambitionen zurückstellt und die notwendige Kontinuität in der Quasifamilie gewährleistet. Das PartnerErsatzmodell, das hier entstanden ist, verbleibt in den geschlechtsspezifischen Arbeitsteilungsmustern, die jeweils ihre spezifischen Machtressourcen reproduzieren. Also: Business as usual?

Diese Fallbeschreibung aus meinen empirischen Untersuchungen (Lutz 2007a, S. 109 ff.) zeigt plastisch, dass finanzielle und relationale Asymmetrien nicht automatisch verschwinden, wenn die Fürsorgearbeit an eine Migrantin weitergereicht wird. In der Studie stellt dieses Beispiel eine einmalige Konstellation dar, die allerdings die Problematik der Umverteilung sehr anschaulich macht: Hier spiegelt sich die fehlende gesellschaftliche Aufwertung der Fürsorgearbeit und ihre geschlechtsspezifische Verstetigung (sie wird an eine Frau weitergereicht). ${ }^{12}$

\section{Fazit}

Obgleich sich die Umverteilung von Fürsorgearbeit auf eine Migrantin in erster Linie im Privathaushalt manifestiert, geht es keineswegs um ein privates, sondern um ein soziales Problem. Zu fragen ist, wie im Zusammenspiel zwischen Staat, Markt und Familie (in)formelle Arrangements entstehen, die jeweils mit spezifischen Geschlechterarrangements, Wohlfahrts- und Migrationsregimen verknüpft sind. Offensichtlich ist das Thema „Migrantinnen in der Fürsorgearbeit" von vielfachen Widersprüchen, Ignoranzen und Tabuisierungsbedürfnissen gekennzeichnet, denn unterschiedliche Akteure vertreten unterschiedliche Interessen:

(1) Die deutsche Zuwanderungspolitik hält weiterhin daran fest, dass eine Öffnung des Arbeitsmarktes im privaten Dienstleistungssektor für nicht-deutsche Arbeitnehmerinnen und Arbeitnehmer nicht notwendig ist. Das Primat des Anwerbestopps, für den im hochqualifizierten und im landwirtschaftlichen Sektor immer wieder Ausnahmen gemacht werden, wird hier als unantastbar betrachtet.

(2) Ökonomen befürworten die Deregulierung des Sektors, um sie als Instrument der nationalen Arbeitsmarktpolitik zur Arbeitsplatzbeschaffung im Niedriglohnsektor zu nutzen.

(3) Pflegeverbände befürchten und wehren sich gegen die Konkurrenz in der Hauspflege, die gerade in den letzten Jahrzehn-

\footnotetext{
11 Die Namen der Interviewten wurden anonymisiert.

12 Weitere theoretische Überlegungen, die sich daraus für die sozialkonstruktivistische Debatte über das Doing Gender ergeben, finden sich in Lutz 2007a, S. $39 f f$.
} 
ten nachhaltig professionalisiert wurde, dementsprechend aufgewertet ist und Preiserhöhungen durchlaufen hat.

(4) Gewerkschaften argumentieren im Sinne nationaler Interessen in ähnlicher Weise wie die Pflegeverbände gegen die Aufweichung der professionalisierten Standards und ihrer Bezahlung; bislang gibt es in Deutschland keine Interessenvertretung für Wanderarbeiterinnen in der Haushaltsarbeit. Einzig in den Wohlfahrtsverbänden der christlichen Kirchen werden nach dem Stellvertreterprinzip Ausbeutung und Missstände angeprangert (Alt 2003).

(5) Für viele Haushalte bleiben Migrantinnen als Haushaltsarbeiterinnen und Pflegekräfte eine vergleichsweise billig und flexibel einsetzbare Alternative zu bürokratischen, kostspieligen institutionalisierten Angeboten.

(6) Für die betroffenen Migrantinnen ist das in Deutschland verdiente Geld eine Ressource, mit deren Hilfe die Existenz und Zukunftsfähigkeit ihrer Familien garantiert werden kann, selbst wenn sie dafür Dequalifizierung und den Verlust von Bürgerrechten in Kauf nehmen müssen. In der Regel wird im Laufe dieser Tätigkeit die Unabhängigkeit von einem Arbeitgeber und der Aufbau eines eigenen Kundennetzes angestrebt (Live-out statt Live-in), um ein Mindestmaß an Autonomie zu erlangen.

(7) Die Herkunftsländer der Migrantinnen sind vor allem an den umfangreichen Remissionen interessiert und bevorzugen die Kategorisierung dieser Frauen als „zeitweise abwesend“ in ihren Statistiken, sodass sie als Emigranten nicht sichtbar werden. Gleichzeitig erleichtern sie die Inanspruchnahme von Kranken- und Sozialversicherung durch diesen Personenkreis im Heimatland (Kempinska 2006).

Insgesamt fehlt es bislang sowohl an formalen Anerkennungsverfahren der durch die Migrantinnen geleisteten Fürsorgearbeit als auch an Legalisierungsanstrengungen für diese Gruppe; in der nationalen Sozialpolitik bleibt die Thematisierung dieses Phänomens tabuisiert. $\mathrm{Zu}$ hoffen ist, dass über Initiativen der Europäischen Wanderarbeitverbände und NGOs, wie etwa „Kein Mensch ist illegal", deutlich gemacht wird, dass die Frage der Fürsorgearbeit nicht mehr im nationalen Kontext zu klären ist, sondern nur unter Berücksichtigung der globalisierten Arbeitsmärkte sinnvolle Lösungen erarbeitet werden können. Auf der Suche nach möglichen Alternativen stellen sich zunächst einmal komplizierte Fragen: Wie können aufenthaltsrechtlich Illegale in ein legalisiertes System (etwa die Dienstleistungspools) übernommen werden, ohne dabei eine Ausweisung zu riskieren? Kann Haushaltsarbeit wirklich ganz normale Erwerbsarbeit sein, wenn sie in einem legalen Arbeitsverhältnis verrichtet wird (Gather et al. 2002)? Würde die Arbeitsübernahme durch eine professionell ausgebildete Frau nicht eine zusätzliche Herausforderung für die beiden Frauen mit sich bringen, die in diesem als weiblich definierten Identitätsraum zusammentreffen? In diesem Zusammenhang fragt Bridget Anderson zu Recht: „Wenn die Haushaltsarbeiterin für ihre Arbeit ausgebildet werden muss, wie kann Haushaltsarbeit dann weiterhin als „die natürliche Kompetenz“ von Frauen im Allgemeinen betrachtet werden? Wenn eine (Haus-)Frau sich durch eine Professionelle ersetzen lässt, beschäftigt sie dann jemand, der „ihren“ eigenen Job besser macht?" (Anderson 2000, S. 169, Übersetzung H. L.). In unseren Interviews wurde immer wieder deutlich, dass Arbeitgeberinnen nicht unbedingt einen „Ersatz“ suchen, sondern eine entlastende Ergänzung; die Grenzen dazwischen sind jedoch fließend.

Da der Privatraum in besonderer Weise der Herstellung von Identität dient, ist anzunehmen, dass die Professionalisierung von Fürsorgearbeit, vielleicht mit Ausnahme der Pflege älterer Menschen, eine tiefe Verunsicherung für einen Kernbereich weiblicher Identitätsarbeit mit sich brächte.

Auf der Suche nach Lösungen muss also das Kernproblem der Fürsorgearbeit, ihre einseitige Verortung als weiblich (vergeschlechtlicht) benannt und das Verhältnis zwischen Fürsorge- und Erwerbsarbeit infrage gestellt werden. Mit anderen Worten: Ohne eine Debatte über die unterschiedliche gesellschaftliche Bewertung von Erwerbs- und Familienarbeit als Ausdruck einer asymmetrischen Geschlechterordnung wird eine Professionalisierungsdebatte nicht auskommen. Manchen mag diese Aufforderung bekannt vorkommen; sie ist bereits vor 30 Jahren in der feministischen Bewegung geäußert worden und kam in der Kampagne „Lohn für Hausarbeit" zum Ausdruck. Es ist anzunehmen, dass die Aktivistinnen von damals nicht antizipieren konnten, dass diese Losung einmal ihre metaphorische Bedeutung verlieren und sich auf sehr eigenwillige Weise zu einem Realphänomen entwickeln würde.

\section{LITERATUR}

Anonymus (2007): Wohin mit Vater? Ein Sohn verzweifelt am Pflegesystem, Frankfurt/Main

Alt, J. (2003): Leben in der Schattenwelt. Problemkomplex "illegale“ Migration. Neue Erkenntnisse zur Lebenssituation "illegaler" Migranten aus München und anderen Orten Deutschlands, Karlsruhe

Andall, J. (2000): Gender, Migration and Domestic Service. The Politics of Black Women in Italy, Aldershot

Andall, J. (2003): Hierarchy and Interdependence: The Emergence of a Service Caste in Europe, in: Dies. (Hrsg.): Gender and Ethnicity in Contemporary Europe, Oxford, S. 39-60
Anderson, B. (2000): Doing the Dirty Work? The Global Politics of Domestic Labour, London/ New York

Anderson, Ph. (2003): „Dass Sie uns nicht vergessen...“. Menschen in der Illegalität in München. Eine empirische Studie im Auftrag der Landeshauptstadt München, München: Sozialreferat, Stelle für Interkulturelle Zusammenarbeit

Bock, G./Duden, B. (1977): Arbeit aus Liebe - Liebe als Arbeit. Zur Entstehung der Hausarbeit im Kapitalismus, in: Gruppe Berliner Dozentinnen (Hrsg.): Frauen und Wissenschaft, Beiträge zur Berliner Sommeruniversität für Frauen, Berlin, S. 118-199 
Bundesministerium für Familie, Senioren, Frauen und Jugend (BMFSFJ) (Hrsg.) 2006: Siebter Familienbericht: Familie zwischen Flexibilität und Verlässlichkeit. Perspektiven für eine lebenslaufbezogene Familienpolitik, Berlin

Cyrus, N. (2004): Aufenthaltsrechtliche Illegalität in Deutschland. Bericht für den Sachverständigenrat für Zuwanderung und Integration, Nürnberg/Berlin

Deutsches Institut für Wirtschaftsforschung (DIW) (2005): Sozialoekonomisches Panel 2005, Berlin

Friese, M./Thiessen, B. (1997): Modellprojekt "Mobiler Haushaltsservice" - ein innovatives Konzept für die Ausbildung und Beschäftigung von Hauswirtschafterinnen, Bremen

Gather, C./Geissler, B./Rerrich, M. (Hrsg.) (2002): Weltmarkt Privathaushalt. Bezahlte Hausarbeit im globalen Wandel, Münster

Gamburd, M. (2000): The Kitchen Spoon's Handle. Transnationalism and Sri Lankan's Housemaids, Ithaka/NY

Hess, S. (2005): Globalisierte Hausarbeit: Au Pair als Migrationsstrategie von Frauen aus Osteuropa, Wiesbaden

Hochschild, A. R. (2000): Global Care Chains and Emotional Surplus Value, in: Giddens, A./Hutton, W. (Hrsg.): On the Edge. Living with Global Capitalism, London, S. 130-146

Holch, Chr. (2006): Die 24-Stunden-Polin, in: Chrismon 11, S. 41-48 Hondagnen-Sotelo, P./Avila E. (1997): I'm here, but I'm there. The meanings of Latina transnational motherhood, in: Gender \& Society 5 , S. 548-571

Kempinska, E. (2006) Recent trends in International Migration. The SOPEMI Report from Poland, Centre for Migration Research, Warsaw Lutz, H. (2007a): Vom Weltmarkt in den Privathaushalt. Die neuen Dienstmädchen im Zeitalter der Globalisierung, Opladen Lutz, H. (Hrsg.) (2007b): Migrant Domestic Work - A European Perspective on a Global Theme, Aldershot (im Erscheinen)

OECD (1999): A Caring World. The New Cocial Policy Agenda, Paris Momsen, J. H. (1999): Maids on the Move, in: Dies. (Hrsg.): Gender, Migration and Domestic Service, London
Parreñas, R. S. (2001): Servants of Globalization. Women, Migration and Domestic Work, Stanford

Parreñas, R. S. (2005): Children of Global Migration. Transnational Families and Gendered Woes, Stanford

Pessar, P./Mahler, S. (2003): Transnational Migration: Bringing Gender in, in: International Migration Review 3, S. 812-846

Rerrich, M. (2006): Die ganze Welt zuhause, Hamburg

Sassen, S. (1998): Überlegungen zu einer feministischen Analyse der globalen Wirtschaft, in: PROKLA 111, S. 199-216

Shinozaki, K. (2003): Geschlechterverhältnisse in der transnationalen Elternschaft. Das Beispiel philippinischer HausarbeiterInnen in Deutschland, in: Beiträge zur feministischen Theorie und Praxis 3, S. 67-85

Sørensen, N. (2005): Migrant Remittances, Development and Gender, Kopenhagen: Dansk Institut for internationale studier

Schupp, J. (2002): Quantitative Verbreitung von Erwerbstätigkeit in privaten Haushalten Deutschlands in: Gather, C./Geissler, B./Rerrich, M. (Hrsg.): Weltmarkt Privathaushalt. Bezahlte Hausarbeit im globalen Wandel, Münster, S. 50-70

Schupp, J./Spieß, K./Wagner, G. (2006): Beschäftigungspotentiale in privaten Haushalten nicht überschätzen, in: DIW Wochenbericht 41, S. $45-52$

Statistisches Bundesamt (2003): Wo bleibt die Zeit? Die Zeitverwendung der Bevölkerung in Deutschland 2001/2, Wiesbaden

Weinkopf, C. (2002): "Es geht auch anders" - reguläre Beschäftigung durch Dienstleistungspools, in: Gather, C./Geissler, B./Rerrich, M. (Hrsg.): Weltmarkt Privathaushalt. Bezahlte Hausarbeit im globalen Wandel,

Münster, S. 154-166

Weinkopf, C. (2003): Förderung haushaltsbezogener Dienstleistungen Sinnvoll, aber kurzfristige Beschäftigungswirklungen nicht überschätzen, in: Vierteljahreshefte zur Wirtschaftsforschung 1, S. 133-147

Zimmermann, K. F. (2003): Beschäftigungspotentiale im Niedriglohnsektor, in: Vierteljahreshefte zur Wirtschaftsforschung 1, S.11-24 